\title{
Ethnic Differences in the Metabolism of Toluene: Comparisons between Korean and Foreign Workers Exposed to Toluene
}

\author{
Ki-Woong Kim, Young Lim Won and Kyung Sun Ko \\ Occupational Safety and Health Research Institute, KOSHA, Ulsan, Korea
}

(Received February 24, 2015; Revised March 19, 2015; Accepted March 23, 2015)

\begin{abstract}
The objectives of this study were to investigate the individual characteristics, lifestyle habits, exposure levels, and genetic diversity of xenobiotic-metabolizing enzymes involved in toluene metabolism in Korean and foreign workers exposed to toluene at a manufacturing plant. This study was conducted to determine the effects of culture or ethnicity on toluene metabolism. The results showed that blood and urinary toluene concentrations were dependent on the level of exposure to toluene. We analyzed the correlation between toluene metabolism and genetic diversity in glutathione S-transferase (GST) (M1), GSTT1, and cytochrome p-450 (CYP) 2E1*5 as well as lifestyle habits (smoking, drinking, and exercise habits). The results revealed significant correlations between toluene metabolism and GSTM1 and GSTT1 genetic diversity, as well as smoking and exercise.
\end{abstract}

Key words: Ethnic differences, Asian, Toluene, Biological monitoring, Genetic polymorphisms

\section{INTRODUCTION}

Korea established the "Act on Foreign Workers' Employment, etc." in 2003, and implemented an employment permit system for foreigners from August 17, 2004. This program has led to an annual increase in the number of foreign residents in Korea, with the number reaching 576,034 in 2013. The number of foreign residents with employment authorization was approximately 549,202, and 91\% (499,036 residents) were reported to be unskilled workers (1). The inability of these unskilled workers to find employment in workplaces that require professional skills, often leads to them being hired in manufacturing industries hiring them, which do not require any particular skills. The majority of manufacturing businesses that hire unskilled workers have work environments with a higher exposure to harmful substances and higher work intensity than businesses requiring specialized skills. Therefore, most of these working environments may be considered as unhealthy. Consequently,

Correspondence to: Ki-Woong Kim, Occupational Health Research Department, Occupational Safety and Health Research Institute, KOSHA, 400, Jongga-ro, Jung-gu, Ulsan 681-230, Korea E-mail: k0810@kosha.net

This is an Open-Access article distributed under the terms of the Creative Commons Attribution Non-Commercial License (http:// creativecommons.org/licenses/by-nc/3.0) which permits unrestricted non-commercial use, distribution, and reproduction in any medium, provided the original work is properly cited. there is an urgent need to assess the effects of such work environments on the health of foreign workers. However, despite this need, proper evaluations are not being carried out on the account of the illegal employment status of most of these workers, as well as communication difficulties, and unconcerned employers. In retrospect, there have been a series of cases of intoxications previously where foreign workers were exposed to harmful chemicals. This exposure resulted in a harmful effects including polyneuropathy caused by $n$-hexane exposure at a liquid crystal display (LCD) parts manufacturer in 2005 (2). In addition, fulminant liver necrosis caused by exposure to dimethylformamide (DMF) occurred at a synthetic leather manufacturing industry in 2006; Stevens-Johnson syndrome caused by exposure to trichloroethylene (TCE) was discovered in a repair factory in 2008, and asthma and toxic hepatitis were reported in 2011 and 2012 (2-4). If the problems mentioned above are not addressed, there is a high risk of future health hazards arising in foreign workers exposed to harmful chemicals. In addition, workplaces that do not demand particular skills, also pose a similar threat of intoxication induced by exposure to harmful chemicals, to Korean workers as well. In particular, toluene (CAS NO., 108-88-3) which is a monocylic aromatic hydrocarbon and widely used in industry as paints, inks, thinner, coatings and etc., is very likely to cause adverse health effects among workers. Absorbed into a human body, toluene is metabolized by cytochrome p-450 (CYP) and glutathione-S-transferase (GST) 
to produce metabolic intermediates and a by-product of reactive oxygen species (ROS) which are toxic to organs such as central nervous system (CNS) and liver. Studies on the health effects of toluene exposure have revealed that toluene mainly effect the CNS (5), causing an increased tendency to sleep, frequent headaches, eye irritation and memory impairment in humans (6). Consequently, time weighted average (TWA) value for toluene was lowered from 100 ppm to $50 \mathrm{ppm}$ in 2007 following many research proving the severity of toxicity of toluene (7). And a no-observedadverse-effect level (NOAEL) and a lowest-observed-effect level (LOAEL) were $20 \mathrm{ppm}$ and $>100 \mathrm{ppm}$, respectively (8).

Harmful chemicals have unique physicochemical characteristics and differ in their route of absorption into the body as well as their target organs. This leads to differences in the manifestations of somatic intoxication. Human toxicity caused by exposure to harmful chemicals depends on the time and concentration of exposure and is closely related to the activity of xenobiotic-metabolizing enzymes. The activity of xenobiotic-metabolizing enzymes is affected by environmental and genetic factors (9). Environmental factors include lifestyle and dietary habits (alcohol, smoking, exercise, etc.) and the presence of disease while genetic factors include ethnicity and differences in the specific gene expression patterns of individuals. These differences directly influence the expression and induction of xenobiotic-metabolizing enzymes (10-13). While the level of exposure is considered a major cause of the intoxications in foreign workers induced by harmful chemical exposure, it is impossible to exclude the influence of lifestyle habits and metabolic sensitivity, due to genetic diversity associated with ethnic differences.

Accordingly, the objectives of this study were to elucidate the influence of individual characteristics, lifestyle habits, exposure levels, and genetic diversity in xenobioticmetabolizing enzymes on toluene metabolism in Korean and foreign workers at manufacturing sites. In addition, we also investigated the influence of culture and ethnicity on toluene metabolism.

\section{MATERIALS AND METHODS}

Study subjects. The subjects included in this study were Korean and foreign male workers exposed to toluene at a printing company that utilizes chemical products. There were 161 subjects consisting of 73 Korean and 88 foreign workers (20 Vietnamese, 24 Sri Lankans, 16 Indonesians, 17 Chinese, and 13 Cambodians). The study was conducted after the consideration and approval of the Institutional Review Board of the Occupational Safety and Health Research Institute (OSHRI). We visited the target workplace and provided the workers with a detailed explanation of the study objectives, methods, protection of personal information, and other matters pertaining to the study protocol. The study commenced after we obtained the consent of workers who were willing to participate in the study. Life habit, general and job characteristics of the subjects were surveyed using self-reported questionnaire and interview.

Measurement of anthropometric parameters and serum biochemistry test. Body mass index (BMI) was measured using body composition analyser (X-SCAN plus II, Jawon Medical, Seoul, Korea), and systolic and diastolic blood pressures were measured using mercury manometer after 10-min rest. Visceral fat thickness (VFT) and subcutaneous fat thickness (SFT) were measured with ultrasonic diagnostic equipment (SonoAce 8800, Medison, Seoul, Korea) using B-mode ultrasound $3.5 \mathrm{MHz}$ oval probe. Blood was collected at 08:00-09:00 AM from workers in fasting state since 10:00 PM previous day. Serum biochemistry including albumin (ALB), alkaline phosphatase (ALP), alanine aminotransferase (ALT), aspartate aminotransferase (AST), gamma-glutamyl transferase (GGT), glucose, triglyceride, total cholesterol, high-density lipoprotein (HDL) and low-density lipoprotein (LDL) cholesterol was performed by automatic biochemistry analyser (COBAS Integra 400, Roch Diagnostic Ltd., Rotkreuz, Switzerland).

Concentrations of toluene in blood and urine. To analyze the concentration of toluene in the blood, $1 \mathrm{~mL}$ of blood was collected from each subject and placed in a $20 \mathrm{~mL}$ headspace injection vial. The stopper was immediately replaced and a gas chromatograph/mass selective detector with a headspace sampler (Hewlett-Packard 7694 headspace sampler/6890 GC/5793 Mass Selective Detector, USA) was used for the determination. The concentration of toluene in urine was analyzed by placing $2.5 \mathrm{~mL}$ of the urine sample in a $20 \mathrm{~mL}$ headspace injection vial containing approximately $1 \mathrm{~g}$ of sodium chloride. The stopper was immediately closed and after saturating the sample with sodium chloride, a gas chromatograph/mass selective detector with a headspace sampler (Hewlett-Packard 7694 headspace sampler/6890 GC/5793 Mass Selective Detector, USA) was used for the determination. A DB-624 (60 $\mathrm{m} \times$ $0.25 \mathrm{~mm}, 1.4 \mu \mathrm{m}$ ) column was used for analysis.

Genotyping of glutathione S-transferase (GST)-linked enzymes and cytochrome P-450 (CYP)2E1*5. The genetic diversity of glutathione S-transferase (GST) M1 and T1 (GSTT1) were analyzed using the method of Shaikh et al. (14) with slight modifications. A total of $10 \mathrm{pM}$ each of the GSTM1 (sense, 5'-GAACTCCCTGAAAAGCTAAAG3' and anti-sense, 5'-GTTGGGCTCAAATATACGGTGG-3') and GSTT1 (sense, 5'-TTCCTTACTGGTCCTCACATCTC$3^{\prime}$ and anti-sense, 5'-TCACCGGATCATGGCCAGCA-3') primers was added to $1 \mu \mathrm{L}$ of the genomic DNA extracted 
(A)

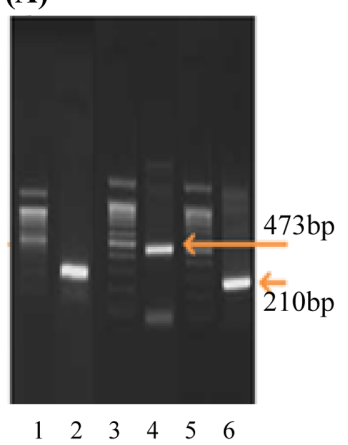

(B)

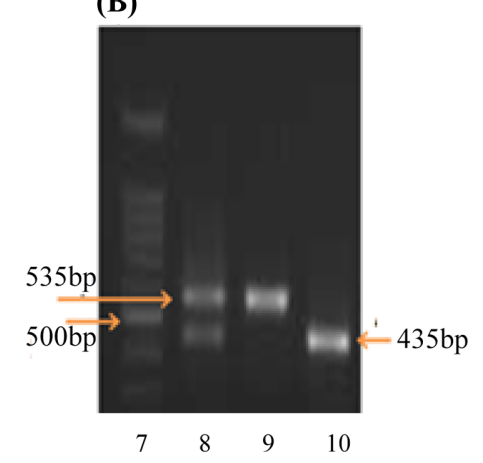

Fig. 1. Analysis of GSTM1, GSTT1 and CYP2E1*5 restriction fragment length polymorphisms (RFLP) by polymerase chain reaction. (A) GSTM1 (210 bp) and GSTT1 (473 bp) (B) CYP2E1*5. c1/c1 (535 bp), c1/c2 (535 and 435 bp) and c2/c2 (435 bp). Lane 1, 6 and 7, size marker; lane 2 and 3, GSTM1 present and null types; lane 4 and 5, GSTT1 present and null types; lane 8, c1/c2; lane $9, \mathrm{c} 1 / \mathrm{c} 1$ and lane $10, \mathrm{c} 2 / \mathrm{c} 2$.

from the blood samples, and then mixed with AccuPowerTM PCR premix (Bioneer Co., Korea) containing $10 \mathrm{mM}$ tris- $\mathrm{HCl}$ (pH 9.0), $40 \mathrm{mM} \mathrm{KCl}, 1.5 \mathrm{mM} \mathrm{MgCl} 2,1 \mathrm{U}$ DNA polymerase, and $1 \mathrm{mM}$ dNTP. The total volume was then made up to $20 \mu \mathrm{L}$ with triple-distilled water, before amplification with a gene amplifier (iCycler, BIO-RAD Co., USA). The amplified product was subjected to electrophoresis on a $1.5 \%$ agarose gel, and the genetic diversity was determined by checking for the presence of bands at 210 and 473 bp (Fig. 1A).
The primers (sense, 5'-CCAGTCGAGTCTACATTGTCA$3^{\prime}$ and anti-sense, 5'-AGACCTCCACATTGACTAGC-3') used in the CYP2E $1 * 5$ genetic diversity analysis were constructed according to the method of Cai et al. (15), and analyzed using the method of Prieto-Castello et al. (16) with slight changes. After mixing $10 \mathrm{mM}$ tris- $\mathrm{HCl}(\mathrm{pH} \mathrm{9.0)}$, $40 \mathrm{mM} \mathrm{KCl}, 1.5 \mathrm{mM} \mathrm{MgCl}, 1 \mathrm{U}$ DNA polymerase, $10 \mathrm{pM}$ of primers, and $1 \mu \mathrm{L}$ of genomic DNA the total volume was made up to $20 \mu \mathrm{L}$ with triple-distilled water, followed by amplification using a gene amplifier (iCycler, BIO-RAD Co., USA). After cutting specific parts of the gene amplification products using the Pst I restriction enzyme, electrophoresis was performed on $2.0 \%$ agarose gel and the gene alleles were confirmed (Fig. 1B).

Statistical analysis. All data were analyzed using the statistics for the social sciences SPSS Version 18 (SPSS Inc., USA). For the comparison of foreign workers based on country, a one-way ANOVA was performed, and correlations between variables were determined using the multiple logistic regression analysis. All results are presented as the mean \pm standard deviation (SD).

\section{RESULTS}

General characteristics of subjects. Based on the data obtained from the questionnaires and direct interviews with the foreign workers, general characteristics such as age, marital status, level of education, smoking and drinking habits, dietary habits, exercise habits, and daily sleep time

Table 1. Categorical characteristics of study subjects

\begin{tabular}{|c|c|c|c|c|c|c|}
\hline Nations & Korean $(n=73)$ & Vietn. $(n=20)$ & SriL. $(n=24)$ & Indon. $(\mathrm{n}=16)$ & Chinese $(n=17)$ & Camb. $(n=13)$ \\
\hline Age (years) & $42.3 \pm 9.6^{\mathrm{c}}$ & $28.5 \pm 4.7^{\mathrm{a}}$ & $29.9 \pm 4.1^{\mathrm{a}}$ & $30.9 \pm 6.2^{\mathrm{a}}$ & $35.1 \pm 7.9^{\mathrm{ab}}$ & $33.9 \pm 6.0^{\mathrm{ab}}$ \\
\hline \multicolumn{7}{|l|}{ Marital status, n (\%) } \\
\hline Single & $12(16.7)^{\mathrm{ab}}$ & $11(55.0)^{\mathrm{ab}}$ & $18(75.0)^{\mathrm{a}}$ & $12(75.0)^{\mathrm{a}}$ & $3(17.6)^{\mathrm{ab}}$ & $4(30.8)^{\mathrm{ab}}$ \\
\hline Married & $61(83.3)$ & $9(45.0)$ & $6(25.0)$ & $4(25.0)$ & $14(82.4)$ & $9(69.2)$ \\
\hline \multicolumn{7}{|l|}{ Smoking habit, n (\%) } \\
\hline Never & $18(24.7)^{\mathrm{ab}}$ & $11(55.0)^{\mathrm{ab}}$ & $18(75.0)^{\mathrm{b}}$ & $8(50.0)^{\mathrm{ab}}$ & $6(35.3)^{\mathrm{ab}}$ & $10(76.9)^{\mathrm{b}}$ \\
\hline Ex-smoker & $22(30.1)$ & $2(10.0)$ & $0(0.0)$ & $1(6.3)$ & $1(5.9)$ & $1(7.7)$ \\
\hline Current & $33(45.2)$ & $7(35.0)$ & $6(25.0)$ & $7(43.7)$ & $10(58.8)$ & $2(15.4)$ \\
\hline \multicolumn{7}{|l|}{ Drinking habit, n (\%) } \\
\hline Never & $19(25.4)^{\mathrm{c}}$ & $14(70.0)^{\mathrm{ab}}$ & $6(25.0)^{\mathrm{ab}}$ & $10(62.5)^{\mathrm{ab}}$ & $5(29.4)^{\mathrm{c}}$ & $5(38.5)^{\mathrm{ab}}$ \\
\hline Ex-drinker & $9(10.4)$ & $2(10.02)$ & $8(33.3)$ & $2(12.5)$ & $2(11.8)$ & $4(30.7)$ \\
\hline Current & $45(64.2)$ & $4(20.0)$ & $10(41.7)$ & $4(25.0)$ & $10(58.8)$ & $4(30.7)$ \\
\hline Food intake & $20.3 \pm 2.9^{\mathrm{a}}$ & $22.4 \pm 2.5^{\mathrm{c}}$ & $18.4 \pm 2.8^{\mathrm{b}}$ & $19.5 \pm 1.8^{\mathrm{a}}$ & $19.6 \pm 2.5^{\mathrm{a}}$ & $20.9 \pm 1.4^{\mathrm{ab}}$ \\
\hline \multicolumn{7}{|l|}{ Regular exercise, n (\%) } \\
\hline Yes & $21(28.8)^{\mathrm{ab}}$ & $9(45.0)^{\mathrm{a}}$ & $4(16.7)^{b}$ & $6(37.5)^{\mathrm{ab}}$ & $7(41.2)^{\mathrm{ab}}$ & $6(46.2)^{\mathrm{a}}$ \\
\hline No & $52(71.2)$ & $11(55.0)$ & $20(83.3)$ & $10(62.5)$ & $10(58.8)$ & $7(53.8)$ \\
\hline Sleeping time (hrs) & $6.9 \pm 0.9^{a}$ & $6.9 \pm 0.7^{\mathrm{a}}$ & $7.0 \pm 0.9^{\mathrm{a}}$ & $6.6 \pm 0.9^{\mathrm{a}}$ & $6.9 \pm 1.3^{\mathrm{a}}$ & $7.5 \pm 1.3^{\mathrm{ab}}$ \\
\hline Working duration (years) & $4.7 \pm 4.8^{\mathrm{a}}$ & $2.5 \pm 2.7^{\mathrm{b}}$ & $3.1 \pm 3.0^{\mathrm{b}}$ & $1.2 \pm 1.1^{\mathrm{b}}$ & $1.7 \pm 1.6^{\mathrm{b}}$ & $2.8 \pm 2.5^{\mathrm{b}}$ \\
\hline Working hours/day & $9.5 \pm 1.8^{\mathrm{a}}$ & $10.1 \pm 1.5^{\mathrm{a}}$ & $10.2 \pm 3.3^{\mathrm{a}}$ & $10.4 \pm 2.9^{\mathrm{a}}$ & $10.1 \pm 2.1^{\mathrm{a}}$ & $11.0 \pm 3.0^{\mathrm{a}}$ \\
\hline
\end{tabular}

Vietn., Vietnamese; SriL., Sri Lankan; Indon., Indonesian; Camb., Cambodian; Upper letters (a, b, c): The same letters are not significantly different (Scheffe test, $p<0.05$ ). 
are presented in Table 1 and classified according to the country of origin. The mean age of the Korean subjects was $42.3 \pm 9.6$ years, which was significantly higher than that of the foreign subjects at $35.1 \pm 7.9,33.9 \pm 6.0,30.9 \pm 6.2$, $29.9 \pm 4.1$, and $28.5 \pm 4.7$ years for the Chinese, Cambodians, Indonesians, Sri Lankans, and Vietnamese, respectively $(p<0.05)$. In terms of marital status, there were a higher number of unmarried Sri Lankan and Indonesia workers $(25 \%$ each, $p<0.05)$. There were fewer smokers among the Sri Lankan (25.0\%) and Cambodian (15.4\%) workers $(p<0.05)$, and a large number of the workers who drank alcohol were Korean (64.2\%) and Chinese (58.8\%, $p<0.05)$. The dietary habits of the Vietnamese and Sri Lankan workers differed from those of other countries. In particular, more Vietnamese $(45.0 \%)$ and Cambodian $(46.2 \%)$ workers participated in regular exercise than Sri Lankan $(16.7 \%)$ workers did $(p<0.05)$. The mean duration of employment was significantly longer for Korean workers at $4.7 \pm 4.8$ years than for workers from other countries $(p<0.05)$. However, there were no differences in daily working hours.

\section{Anthropometric and clinical characteristics of sub-} jects. The anthropometric measurements and clinical parameters evaluated in the subjects are shown in Table 2. There was no significant difference in BMI between workers from different countries. However, the body fat percentage of workers from Indonesia was significantly lower $(16.5 \pm 4.6 \%)$ than workers from other countries $(p<0.05)$. The systolic and diastolic blood pressure, as well as subcutaneous and visceral fat, showed no differences. The results of the blood biochemical tests showed that the average concentration of high-density lipoprotein (HDL) cholesterol was significantly lower in Cambodian workers (37.5 \pm 9.5 $\mathrm{mg} / \mathrm{dl}$ ) than in other foreign workers. However, there was no difference in the levels of albumin, ALP, ALT, AST, GGT, blood sugar, LDL-cholesterol, and triglycerides between foreign workers from other countries.

Table 2. Anthropometric and clinical characteristics of study subjects

\begin{tabular}{|c|c|c|c|c|c|c|}
\hline Variables & $\begin{array}{l}\text { Korean } \\
(\mathrm{n}=73)\end{array}$ & $\begin{array}{l}\text { Vietnamese } \\
(\mathrm{n}=20)\end{array}$ & $\begin{array}{l}\text { Sri Lankan } \\
(\mathrm{n}=24)\end{array}$ & $\begin{array}{l}\text { Indonesian } \\
(\mathrm{n}=16)\end{array}$ & $\begin{array}{l}\text { Chinese } \\
(n=17)\end{array}$ & $\begin{array}{c}\text { Cambodian } \\
(\mathrm{n}=13)\end{array}$ \\
\hline BMI $\left(\mathrm{kg} / \mathrm{m}^{2}\right)$ & $24.1 \pm 3.2^{\mathrm{a}}$ & $21.4 \pm 3.0^{\mathrm{a}}$ & $22.2 \pm 3.1^{\mathrm{a}}$ & $22.1 \pm 1.7^{\mathrm{a}}$ & $23.8 \pm 3.4^{\mathrm{a}}$ & $23.2 \pm 2.4^{\mathrm{a}}$ \\
\hline $\mathrm{BF} \%$ & $22.4 \pm 5.1^{\mathrm{ab}}$ & $17.8 \pm 4.9^{\mathrm{ab}}$ & $20.4 \pm 6.2^{\mathrm{ab}}$ & $16.5 \pm 4.6^{\mathrm{a}}$ & $21.9 \pm 5.5^{\mathrm{ab}}$ & $20.5 \pm 5.7^{\mathrm{ab}}$ \\
\hline SBP (mm Hg) & $135.9 \pm 17.5^{\mathrm{a}}$ & $122.3 \pm 15.8^{\mathrm{a}}$ & $122.9 \pm 13.1^{\mathrm{a}}$ & $120.3 \pm 11.6^{\mathrm{a}}$ & $129.9 \pm 10.8^{\mathrm{a}}$ & $125.2 \pm 12.9^{\mathrm{a}}$ \\
\hline DBP (mm Hg) & $79.1 \pm 11.8^{\mathrm{a}}$ & $68.5 \pm 10.6^{\mathrm{a}}$ & $69.3 \pm 7.9^{\mathrm{a}}$ & $67.3 \pm 7.3^{\mathrm{a}}$ & $74.6 \pm 7.3^{\mathrm{a}}$ & $71.4 \pm 8.5^{\mathrm{a}}$ \\
\hline $\mathrm{SFT}(\mathrm{cm})$ & $1.6 \pm 0.6^{\mathrm{a}}$ & $1.1 \pm 0.3^{\mathrm{a}}$ & $1.5 \pm 0.7^{\mathrm{a}}$ & $1.0 \pm 0.0^{\mathrm{a}}$ & $1.4 \pm 0.5^{\mathrm{a}}$ & $1.2 \pm 0.4^{\mathrm{a}}$ \\
\hline VFT $(\mathrm{cm})$ & $3.4 \pm 1.3^{\mathrm{ab}}$ & $3.1 \pm 1.1^{\mathrm{ab}}$ & $3.8 \pm 1.3^{\mathrm{ab}}$ & $3.0 \pm 1.1^{\mathrm{ab}}$ & $4.0 \pm 1.5^{\mathrm{ab}}$ & $3.9 \pm 0.9^{\mathrm{ab}}$ \\
\hline $\operatorname{ALB}(\mathrm{g} / \mathrm{dL})$ & $4.8 \pm 0.3^{\mathrm{a}}$ & $4.7 \pm 0.3^{\mathrm{a}}$ & $4.7 \pm 0.3^{\mathrm{a}}$ & $4.5 \pm 0.5^{\mathrm{a}}$ & $4.6 \pm 0.3^{\mathrm{a}}$ & $4.7 \pm 0.2^{\mathrm{a}}$ \\
\hline $\operatorname{ALP}(\mathrm{IU} / \mathrm{L})$ & $68 \pm 20.2^{\mathrm{a}}$ & $67.2 \pm 15.7^{\mathrm{a}}$ & $74.2 \pm 29.5^{\mathrm{a}}$ & $62.6 \pm 15.9^{\mathrm{a}}$ & $67.3 \pm 19.5^{\mathrm{a}}$ & $70.2 \pm 21.4^{\mathrm{a}}$ \\
\hline ALT (IU/L) & $23.2 \pm 21.1^{\mathrm{a}}$ & $20.5 \pm 15.6^{\mathrm{a}}$ & $30.6 \pm 25.6^{\mathrm{a}}$ & $16.2 \pm 8.2^{\mathrm{a}}$ & $19.2 \pm 7.9^{\mathrm{a}}$ & $31.4 \pm 20.2^{\mathrm{a}}$ \\
\hline AST (IU/L) & $22 \pm 12.2^{\mathrm{a}}$ & $20.9 \pm 5.2^{\mathrm{a}}$ & $25.2 \pm 9.1^{\mathrm{a}}$ & $18.7 \pm 5.8^{\mathrm{a}}$ & $19.0 \pm 4.5^{\mathrm{a}}$ & $23.5 \pm 7.1^{\mathrm{a}}$ \\
\hline GGT (IU/L) & $31.9 \pm 36.2^{\mathrm{a}}$ & $22.3 \pm 13.3^{\mathrm{a}}$ & $33.1 \pm 31.2^{\mathrm{a}}$ & $19.5 \pm 14.7^{\mathrm{a}}$ & $32.4 \pm 25.2^{\mathrm{a}}$ & $35 \pm 27.3^{\mathrm{a}}$ \\
\hline GLU (mg/dL) & $103.2 \pm 31.5^{\mathrm{a}}$ & $90.5 \pm 19.8^{\mathrm{a}}$ & $93.3 \pm 17.8^{\mathrm{a}}$ & $83.4 \pm 9.4^{\mathrm{a}}$ & $90.5 \pm 14.8^{\mathrm{a}}$ & $76.6 \pm 5.4^{\mathrm{a}}$ \\
\hline T-Chol. (mg/dL) & $185.3 \pm 31.9^{\mathrm{a}}$ & $165.7 \pm 32.3^{\mathrm{a}}$ & $182.2 \pm 32.1^{\mathrm{a}}$ & $167.4 \pm 34.6^{\mathrm{a}}$ & $169.1 \pm 22.3^{\mathrm{a}}$ & $168 \pm 31.5^{\mathrm{a}}$ \\
\hline HDL-Chol. (mg/dL) & $48.7 \pm 5.2^{\mathrm{bc}}$ & $47.6 \pm 14.3^{\mathrm{bc}}$ & $46.7 \pm 18.1^{\mathrm{bc}}$ & $47.1 \pm 9.6^{\mathrm{bc}}$ & $45.7 \pm 14.9^{\mathrm{bc}}$ & $37.5 \pm 9.5^{\mathrm{c}}$ \\
\hline LDL-Chol. (mg/dL) & $106.5 \pm 36.7^{\mathrm{a}}$ & $104 \pm 25.7^{\mathrm{a}}$ & $116.4 \pm 29.8^{\mathrm{a}}$ & $106.2 \pm 29.4^{\mathrm{a}}$ & $96.2 \pm 31.4^{\mathrm{a}}$ & $109.9 \pm 26.3^{\mathrm{a}}$ \\
\hline Triglyceride (mg/dL) & $198.0 \pm 177.5^{\mathrm{a}}$ & $116.8 \pm 70.3^{\mathrm{a}}$ & $132.8 \pm 59.3^{\mathrm{a}}$ & $105.6 \pm 30.5^{\mathrm{a}}$ & $188.1 \pm 196.9^{\mathrm{a}}$ & $137.8 \pm 56.1^{\mathrm{a}}$ \\
\hline
\end{tabular}

BMI, body mass index; BF\%, body fat \%; SBP, systolic blood pressure; DBP, diastolic blood pressure; SFT, subcutaneous fat thickness; VFT, visceral fat thickness; ALB, albumin; ALP, alkaline phosphatase; ALT, alanine aminotransferase; AST, aspartate aminotransferase; GGT, gammaglutamyl transferase; GLU, glucose; T-Chol, total cholesterol; HDL-Chol, high density lipoprotein-cholesterol; LDL-Chol, low density lipoprotein-cholesterol; Triglyceride (mg/dl); Upper letters $(a, b, c)$ : The same letters are not significantly different (Scheffe test, $p<0.05)$.

Table 3. Comparisons of blood and urinary toluene levels between Korean and foreign workers exposed to toluene

\begin{tabular}{|c|c|c|c|c|c|c|}
\hline Toluene & $\begin{array}{l}\text { Ambient } \\
(\mathrm{ppm})\end{array}$ & $\begin{array}{c}\text { Blood (B) } \\
(\mu \mathrm{g} / \mathrm{L})\end{array}$ & $\begin{array}{c}\text { Urine }(\mathrm{U}) \\
(\mu \mathrm{g} / \mathrm{L})\end{array}$ & $\begin{array}{c}\mathrm{HA} \\
(\mathrm{g} / \mathrm{g} \mathrm{Cr})\end{array}$ & $\mathrm{U} / \mathrm{B}$ ratio ( $\beta$ value, $\mathrm{R}^{2}$ value $)$ & $\begin{array}{l}\text { Scheffe test } \\
\text { U/B ratio }\end{array}$ \\
\hline Korean (A) & $2.12 \pm 6.72^{\mathrm{a}}$ & $86.5 \pm 141.4^{\mathrm{a}}$ & $25.6 \pm 65.3^{\mathrm{a}}$ & $0.25 \pm 0.17^{\mathrm{a}}$ & $23.8 \pm 39.8\left(\beta=0.277, R^{2}=0.098\right)$ & $>\mathrm{B}$ \\
\hline Vietnamese (B) & $1.02 \pm 2.46^{\mathrm{a}}$ & $103.3 \pm 138.2^{\mathrm{a}}$ & $30.4 \pm 49.2^{\mathrm{a}}$ & $0.18 \pm 0.16^{\mathrm{a}}$ & $15.7 \pm 21.1\left(\beta=1.381 \mathrm{x}, \mathrm{R}^{2}=0.268\right)$ & $<\mathrm{A},<\mathrm{C},<\mathrm{D},<\mathrm{F}$ \\
\hline Sri Lankan (C) & $10.1 \pm 26.9^{\mathrm{a}}$ & $525.8 \pm 901.4^{\mathrm{a}}$ & $106.9 \pm 273.0^{\mathrm{a}}$ & $0.42 \pm 0.84^{\mathrm{a}}$ & $21.2 \pm 31.7\left(\beta=0.152 \mathrm{x}, \mathrm{R}^{2}=0.455\right)$ & $>\mathrm{B}$ \\
\hline Indonesian (D) & $14.2 \pm 20.3^{\mathrm{a}}$ & $791.6 \pm 830.0^{\mathrm{a}}$ & $276.7 \pm 283.8^{a}$ & $0.82 \pm 0.57^{\mathrm{b}}$ & $50.6 \pm 57.4\left(\beta=0.247 x, R^{2}=0.297\right)$ & $>\mathrm{A}$ \\
\hline Chinese (E) & $10.4 \pm 18.3^{\mathrm{a}}$ & $682.4 \pm 803.7^{\mathrm{a}}$ & $289.6 \pm 799.2^{\mathrm{a}}$ & $0.72 \pm 1.24^{\mathrm{a}}$ & $36.5 \pm 54.5\left(\beta=0.428 x, R^{2}=0.573\right)$ & - \\
\hline Cambodian $(\mathrm{F})$ & $32.1 \pm 15.2^{\mathrm{b}}$ & $1023 \pm 965.1^{\mathrm{a}}$ & $908.1 \pm 998.1^{\mathrm{b}}$ & $0.79 \pm 0.58^{\mathrm{b}}$ & $122 \pm 149\left(\beta=0.784 x, R^{2}=0.093\right)$ & $>\mathrm{B}$ \\
\hline
\end{tabular}

$\mathrm{HA}$, hippuric acid. Upper letters (a, b): The same letters are not significantly different (Scheffe test, $p<0.05)$. 
Concentrations of toluene in blood and urine and urinary hippuric acid. The level of toluene exposure and concentration in the blood and urine, urinary toluene/blood toluene ratio (U/B ratio), and urinary hippuric acid (HA) concentrations are shown in Table 3. The level of toluene exposure and concentration in the urine were significantly higher in Cambodian workers $(32.1 \pm 15.2 \mathrm{ppm}$ and $908.1 \pm$ $998.1 \mu \mathrm{g} / \mathrm{L}$, respectively) than in workers from other countries $(p<0.05)$. However, there were no differences in the concentrations of toluene or its metabolite HA in the blood. The U/B ratio was significantly lower in Vietnamese $(15.7 \pm 21.1)$ and Sri Lankan $(21.2 \pm 31.7)$ workers, and significantly higher in Indonesian $(50.6 \pm 57.4)$ and Cambodian $(122 \pm 149)$ workers $(p<0.05)$. The HA concentration was significantly higher in Indonesian $(0.82 \pm 0.57 \mathrm{~g} / \mathrm{g}$ creatinine $)$ and Cambodian $(0.79 \pm 0.58 \mathrm{~g} / \mathrm{g}$ creatinine $)$ workers.

Genetic polymorphisms of GSTM1, GSTT1, and CYP2E1*5. The genetic diversity of GSTM1, GSTT1, and CYP2E1*5 enzymes in the workers was analyzed and the results are displayed in Table 4 . Firstly, the allelic frequency of the GSTM1 present (GSTM1p, $210 \mathrm{bp}$ ) and GSTM null (GSTM1n) genotypes in Korean workers was 0.44 and 0.56, respectively, while Sri Lankan and Cambodian workers had similar distributions of both genes at 0.58 and 0.42 , and 0.54 and 0.46 , respectively. Conversely, the Vietnamese, (0.15 and 0.85), Indonesian (0.25 and 0.75$)$, and Chinese (0.71 and 0.29) workers showed differences in

Table 4. Genotypes of the GSTM1, GSTT1 and CYP2E1*5 in study subjects

\begin{tabular}{|c|c|c|c|c|c|c|}
\hline & $\begin{array}{l}\text { Korean } \\
(n=73)\end{array}$ & $\begin{array}{l}\text { Vietnamese } \\
(\mathrm{n}=20)\end{array}$ & $\begin{array}{l}\text { Sri Lankan } \\
(\mathrm{n}=24)\end{array}$ & $\begin{array}{l}\text { Indonesian } \\
(\mathrm{n}=16)\end{array}$ & $\begin{array}{l}\text { Chinese } \\
(n=17)\end{array}$ & $\begin{array}{c}\text { Cambodian } \\
(\mathrm{n}=13)\end{array}$ \\
\hline \multicolumn{7}{|l|}{ GSTM1, n (\%) } \\
\hline Present & $32(43.8)$ & $3(15.0)$ & $14(58.3)$ & $4(25.0)$ & $12(70.6)$ & $7(53.8)$ \\
\hline Null & $41(56.2)$ & $17(85.0)$ & $10(41.7)$ & $12(75.0)$ & $5(29.4)$ & $6(46.2)$ \\
\hline \multirow{2}{*}{ Allele Freq. } & $\mathrm{P}=0.44$ & $P=0.15$ & $\mathrm{P}=0.58$ & $\mathrm{P}=0.25$ & $\mathrm{P}=0.71$ & $\mathrm{P}=0.54$ \\
\hline & $\mathrm{N}=0.56$ & $\mathrm{~N}=0.85$ & $\mathrm{~N}=0.42$ & $\mathrm{~N}=0.75$ & $\mathrm{~N}=0.29$ & $\mathrm{~N}=0.46$ \\
\hline \multicolumn{7}{|l|}{ GSTT1, n (\%) } \\
\hline Present & $32(43.8)$ & $10(50.0)$ & $17(70.8)$ & $12(75.0)$ & $3(17.6)$ & $6(46.1)$ \\
\hline Null & $41(56.2)$ & $10(50.0)$ & $7(29.2)$ & $4(25.0)$ & $14(82.4)$ & $7(53.9)$ \\
\hline \multirow{2}{*}{ Allele Freq. } & $P=0.44$ & $P=0.50$ & $\mathrm{P}=0.71$ & $P=0.75$ & $P=0.18$ & $P=0.46$ \\
\hline & $\mathrm{N}=0.56$ & $\mathrm{~N}=0.50$ & $\mathrm{~N}=0.29$ & $\mathrm{~N}=0.25$ & $\mathrm{~N}=0.82$ & $\mathrm{~N}=0.54$ \\
\hline \multicolumn{7}{|c|}{ CYP2E5, n (\%) } \\
\hline $\mathrm{C} 1 / \mathrm{C} 1$ & $42(57.5)$ & $11(55.0)$ & $24(100.0)$ & $11(68.7)$ & $9(52.9)$ & $10(76.9)$ \\
\hline $\mathrm{C} 1 / \mathrm{C} 2$ & $1(1.4)$ & $0(0.0)$ & $0(0.0)$ & $0(0.0)$ & $2(11.8)$ & $0(0.0)$ \\
\hline $\mathrm{C} 2 / \mathrm{C} 2$ & $29(41.1)$ & $9(45.0)$ & $0(0.0)$ & $5(31.3)$ & $6(35.3)$ & $3(23.1)$ \\
\hline \multirow{2}{*}{ Allele Freq. } & $\mathrm{C} 1=0.59$ & $\mathrm{C} 1=0.55$ & $\mathrm{C} 1=1.00$ & $\mathrm{C} 1=0.69$ & $\mathrm{C} 1=0.59$ & $\mathrm{C} 1=0.77$ \\
\hline & $\mathrm{C} 2=0.41$ & $\mathrm{C} 2=0.45$ & $\mathrm{C} 2=0.00$ & $\mathrm{C} 2=0.31$ & $\mathrm{C} 2=0.41$ & $\mathrm{C} 2=0.23$ \\
\hline
\end{tabular}

Table 5. Correlation matrix adjusted age, life habit between individual effect factors related with toluene metabolism

\begin{tabular}{|c|c|c|c|c|c|c|c|c|c|c|c|c|}
\hline \multirow{4}{*}{$\begin{array}{l}\text { Independent } \\
\text { variables }\end{array}$} & \multicolumn{12}{|c|}{ Dependent variables } \\
\hline & \multicolumn{12}{|c|}{ Genetic factors } \\
\hline & \multicolumn{4}{|c|}{ GSTM1 } & \multicolumn{4}{|c|}{ GSTT1 } & \multicolumn{4}{|c|}{ CYP2E5 } \\
\hline & $\beta$ value & S.E. & OR & $\mathrm{p}$ value & $\beta$ value & S.E. & OR & $\mathrm{p}$ value & $\beta$ value & S.E. & OR & $p$ value \\
\hline Urine/Blood & -0.005 & 0.005 & 0.995 & 0.313 & 0.013 & 0.006 & 1.013 & 0.024 & 0.000 & 0.007 & 1.000 & 0.976 \\
\hline HA/Blood & 3.554 & 2.036 & 34.966 & 0.081 & -0.517 & 1.560 & 0.596 & 0.740 & -0.252 & 1.436 & 0.777 & 0.861 \\
\hline \multirow[t]{4}{*}{ HA/Urine } & -0.121 & 0.193 & 0.886 & 0.529 & -0.184 & 0.231 & 0.832 & 0.427 & 0.106 & 0.194 & 1.112 & 0.584 \\
\hline & \multicolumn{12}{|c|}{ Life habit } \\
\hline & \multicolumn{4}{|c|}{ Smoking } & \multicolumn{4}{|c|}{ Drinking } & \multicolumn{4}{|c|}{ Exercise } \\
\hline & $\beta$ value & S.E. & OR & $\mathrm{p}$ value & $\beta$ value & S.E. & OR & $\mathrm{p}$ value & $\beta$ value & S.E. & OR & $\mathrm{p}$ value \\
\hline Urine/Blood & -0.026 & 0.011 & 0.974 & 0.021 & 0.001 & 0.005 & 1.001 & 0.883 & -0.013 & 0.006 & 0.987 & 0.035 \\
\hline HA/Blood & 5.076 & 2.533 & 160.200 & 0.045 & -0.447 & 1.524 & 0.640 & 0.770 & 3.186 & 2.476 & 24.181 & 0.198 \\
\hline HA/Urine & -4.000 & 0.266 & 0.670 & 0.133 & -0.026 & 0.186 & 0.974 & 0.887 & -0.121 & 0.205 & 0.886 & 0.553 \\
\hline
\end{tabular}

HA, hippuric acid. OR, odds ratio. 
the allelic frequencies. However, the statistical significance could not be verified because of the small number of subjects in the study. The frequency of the GSTT1 present (GSTT1p, $473 \mathrm{bp)}$ and GSSTT1 null (GSTT1n) alleles were similarly distributed in Korean ( 0.44 vs 0.56$)$, Vietnamese $(0.50$ vs 0.50$)$ and Cambodian workers $(0.46$ vs $0.54)$. However, this distribution differed from the allelic frequency of workers from other countries. The CYP2E1*5 gene has several genotypes including the $\mathrm{cl} / \mathrm{c} 1(535 \mathrm{bp})$, $\mathrm{c} 1 / \mathrm{c} 2$ (535 bp and $435 \mathrm{bp}$ ), and c2/c2 (435 bp). For the Korean workers, the frequencies of the $\mathrm{c} 1$ and $\mathrm{c} 2$ alleles were 0.59 and 0.41 , respectively, which was different from those of the Sri Lankan (1.0 and 0.0) and Cambodian (0.77 and 0.23 ) workers.

Interrelationship between the factors affecting toluene metabolism. In order to determine the factors that affect toluene metabolism, we first corrected the data for age. We then performed a multiple logistic regression analysis with the genetic factors (GSTM1, GSTT1, and CYP2E1*5 genetic diversity) and lifestyle (smoking, alcohol consumption, and exercise) as dependent variables, and the U/B, HA/blood toluene, and HA/urinary toluene ratios as independent variables. The results displayed in Table 5 show that GSTM1 slightly correlated with the HA/blood ratio (odds ratio $=34.966, p=0.081$ ), while GSTT1 correlated with the $\mathrm{U} / \mathrm{B}$ ratio (odds ratio $=1.013, p=0.024$ ). Among the lifestyle habits, smoking correlated with the $\mathrm{U} / \mathrm{B}$ ratio (odds ratio $=0.974, p=0.021$ ) and HA/Blood toluene ratio (odds ratio $=160.2, p=0.045$ ), while exercise correlated with $\mathrm{U} / \mathrm{B}$ ratio (odds ratio $=0.987, p=0.035$ ).

\section{DISCUSSION}

The external environment affects all organisms, including humans. Organisms including humans, that adequately adapt to their environments survive, while partial adaptation leads to either extinction or metamorphosis. In addition, organisms that have adapted to their external environment over a long period acquire homogeneity, and form populations with similar genetic and somatic constituents (17). Accordingly, nations and ethnicities show cultural and genetic differences because of the differences in their external environments. These differences cause changes in the physiological homeostatic factors, resulting in sensitivity to specific diseases affecting the metabolism of harmful environmental substances $(18,19)$. In particular, xenobioticmetabolizing enzymes are responsible for metabolizing absorbed foreign substances $(20,21)$. However, the xenobiotic-metabolizing capacity is determined by enzymatic activity, which is greatly influenced by genes $(20,21)$. Consequently, the objective of this study was to investigate whether the intoxication experienced by foreign workers exposed to harmful chemicals was attributable to the levels of exposure, or whether it was because of the metabolic sensitivity resulting from human ecological and geographical differences. The results of the study showed differences in age as well as smoking, drinking, and exercise habits, etc., between workers from different countries. The only significant difference discovered following the determination of serum biochemical parameters was that HDL cholesterol concentration was lower in Cambodian workers. The blood and urinary toluene concentrations were dependent on the level of toluene exposure. The metabolism of toluene showed national differences, but we could not verify the statistical correlation because of the small number of subjects in the study. In addition, a multiple logistic regression analysis was conducted on the data obtained from all the workers. For the analysis, the genetic alleles and lifestyle habits (smoking, alcohol consumption, and exercise) were designated as variables and their correlations with toluene metabolism were analyzed. The results showed significant correlations with GSTM1 and GSTT1 genetic diversity, smoking, and exercise. The monocyclic aromatic hydrocarbon (MAH) toluene affects the liver, immune system, and central nervous system (6). Following absorption into the body, toluene undergoes modification to a charged molecule (epoxidation, hydroxylation) during the first step of metabolism (phase I reaction). It is subsequently excreted from the body following a phase II reaction (conjugation). The phase I reaction mostly involves CYP, while the phase II reaction involves GST $(6,22)$. In addition, because the expression of CYP and GST differs according to species, gender, and environmental substances, there have been numerous studies in humans, animals, and microorganisms that have revealed their subfamilies, roles, and genetic structure $(20,23)$. CYP2E1 was the most active in forming benzyl alcohol and genotypes of it is involved in toluene metabolism (24). GST was also involved in toluene metabolism, Mörk et al. (25) reported that the metabolism of toluene was not affected by GSTT1 genotypes.

In this study, no correlation was found between toluene metabolism and the CYP2E1*5 gene allele. However, the GSTM1 and GSTT1 allelic frequencies correlated with the $\mathrm{U} / \mathrm{B}$ and HA/blood toluene ratios. These results are believed to show that the phase II reaction, which involve GST enzymes determine toluene metabolism. Kawamoto et al. (26) reported that the metabolic sensitivity for toluene depended on the CYP2E1 allele, which differs from our results. This difference is likely due to the differences in the exposure levels and the number of subjects between both studies. In a review, Landi (20) reported a difference in the allele frequency of the GST gene based on nationality (ethnicity).

In our study, ethnic-dependent differences in genetic diversity could not be confirmed because the number of workers for each country included was small. However, combining our results with those of Landi (20) revealed an 
apparent difference in toluene metabolism associated with ethnicity. Lifestyle habits (smoking and drinking, dietary, exercise, etc.,) also have a large influence on toluene metabolism. Rather than a direct influence on toluene metabolism, lifestyle habits appear to influence the process of toluene metabolism by affecting the activity of enzymes involved in its metabolism. In relation to dietary habits, Akbay et al. (27) reported that high-fat consumption lowers the activity of GST and glutathione reductase in the liver, while smoking and alcohol consumption also affected CYP and GST activity $(28,29)$.

Our results showed that smoking habits correlated with the HA/blood toluene ratio, while exercise habits correlated with the U/B ratio. As mentioned above, these results appear to reveal an effect on the enzymes involved in toluene metabolism. In addition, the lack of correlation between toluene metabolism and dietary habits is thought to be because the subjects have lived in Korea for over a year, usually in corporate boarding houses and, therefore, have consumed the same kind of food as the Korean workers. The small number of subjects per country limits this study. Accordingly, future studies focused on addressing this limitation will be conducted continually, and used in facilitating the protection of the health of foreign workers.

\section{ACKNOWLEDGMENTS}

This study was supported by the intramural research fund of the Occupational Safety and Health Research Institute (OSHRI).

\section{REFERENCES}

1. Korea Immigration Service. (2013) 2013 Annual report for immigration immigration policy. Ministry of Justice, Korea.

2. Kim, K.W. (2010) Human ecological study on the susceptibility and metabolism of styrene and toluene in foreign workers. Occupational Safety \& Heath Res. Inst. Ann. Research Rep., Korea, pp. 1-43.

3. Occupational Safety and Health Research Institute. (2012) 2011 Diagnosis cases for occupational disease. Occupational Safety \& Heath Res. Inst. Ann. Research Rep., Korea, pp. 1011.

4. Occupational Safety and Health Research Institute. (2013) 2012 Diagnosis cases for occupational disease. Occupational Safety \& Heath Res. Inst. Ann. Research Rep., Korea, pp. 2425.

5. Win-Shwe, T.T. and Fujimaki, H. (2010) Neurotoxicity of toluene. Toxicol. Lett., 198, 93-99.

6. International Programme on Chemical Safety (IPCS). (1985) Toluene. Environmental Health Criteria 52, World Health Organization, Geneva, pp. 83-100.

7. Ministry of Employment and Labor (MOEL). (2013) Notification 2013-38 of the Ministry of Employment and Labor.

8. Eller, N., Netterstrom, B. and Laursen, P. (1999) Risk of chronic effects on the central nervous system at low toluene exposure. Occup. Med., 49, 389-395.

9. Kim, K.W., Shinetugs, B., Heo, K.H., Won, Y.L., Lkhagwasuren, T., Chang, S.K. and Paik, S.G. (2009) Polymorphisms of alcohol metabolizing enzymes and cytochrome P4502E1 genes in Mongolian population. Genes Genomics, 31, 377-385.

10. Brigelius-Flohé, R. (2005) Induction of drug metabolizing enzymes by vitamin E. J. Plant Physiol., 162, 797-802.

11. Moon, Y.J., Wang, X. and Morris, M.E. (2006) Dietary flavonoids: Effects on xenobiotic and carcinogen metabolism. Toxicol. In Vitro, 20, 187-210.

12. Fujihara, J., Yasuda, T., Iida, R., Takatsuka, H., Fujii, Y. and Takeshita, H. (2009) Cytochrome P4501A1, glutathione Stransferase M1 and T1 polymorphisms in Ovambos and Mongolians. Leg. Med., 11 Suppl 1, S408-410.

13. Zordoky, B.N. and El-Kadi, A.O. (2010) Effect of cytochrome P450 polymorphism on arachidonic acid metabolism and their impact on cardiovascular disease. Pharmacol. Ther., 125, 446-463.

14. Shaikh, R.S., Amir, M., Masood, A.I., Sohail, A., Athar, H.U., Siraj, S., Ali, M. and Anjam, M.S. (2010) Frequency distribution of GSTM1 and GSTT1 null allele in Parkistani population and risk of disease incidence. Environ. Toxicol. Pharmacol., 30, 76-79.

15. Cai, L., Zheng, Z.L. and Zhang, Z.F. (2005) Cytochrome p4502E1 polymorphisms and the risk of gastric cardiac cancer. World J. Gastroenterol., 11, 1867-1871.

16. Prieto-Castelló, M.J., Cardona, A., Marhuenda, D., Roei, J.M. and Corno, A. (2010) Use of the CYP2E1 genotype and phenotype for the biological monitoring of occupational exposure to styrene. Toxicol. Lett., 192, 34-39.

17. Manrubia, S.C. (2012) Modelling viral evolution and adaptation: challenges and rewards. Curr. Opin. Virol., 2, 531-537.

18. Wulan, S.N., Weaterterp, K.R. and Plasqui, G. (2010) Ethnic difference in body composition and the associated metabolic profile: a comparative study between Asians and Caucasians. Maturitas, 65, 315-319.

19. Choi, S.E., Liu, M., Palaniappan, L.P., Wang, E.J. and Wong, N.D. (2013) Gender and ethnic differences in the prevalence of type 2 diabetes among Asian subgroups in California. $J$. Diabetes Complications, 27, 429-435.

20. Landi, S. (2000) Mammalian class theta GST and differential susceptibility to carcinogens: a review. Mutat. Res., 463, 247283.

21. Zanger, U.M. and Schwab, M. (2013) Cytochrome P450 enzymes in drug metabolism: regulation of gene expression, enzyme activities, and impact of genetic variation. Pharmacol. Ther., 138, 103-141.

22. Cohr, K.H. and Stokholm, J. (1979) Toluene. a toxicology review. Scand. J. Work Environ. Health, 5, 71-90.

23. Kalsotra, A. and Strobel, H.W. (2006) Cytochrome P450 4F subfamily: At the crossroads of eicosanoid and drug metabolism. Pharmacol. Ther., 112, 589-611.

24. Nakajima, T., Wang, R.S., Elovaara, E., Gonzalez, F.J., Gelboin, H.V., Raunio, H., Pelkonen, O., Vainio, H. and Aoyama, T. (1997) Toluene metabolism by cDNA-expressed human hepatic cytochrome P450. Biochem. Pharmacol., 53, 271-277.

25. Mörk, A.K., Jonsson, F. and Johanson, G. (2014) Adjustment factors for toluene, styrene and methyl chloride by population 
modeling of toxicokinetic variability. Regul. Toxicol. Pharmacol., 69, 78-90.

26. Kawamoto, T., Koga, M., Murata, K., Matsuda, S. and Kodama, Y. (1995) Effects of ALDH2, CYP1A1, and CYP2E1 genetic polymorphisms and smoking and drinking habits on toluene metabolism in humans. Toxicol. Appl. Pharmacol., 133, 295304.

27. Akbay, E., Ulusu, N.N., Töröner, F., Ayvaz, G., Taneri, F., Aktürk, M., Arslan, M. and Karasu, C. (2004) Effects of rosiglitazone treatment on the pentose phosphate pathway and glutathione-dependent enzymes in liver and kidney of rats fed a high-fat diet. Curr. Ther. Res., 65, 79-89.
28. Eke, B.C., Vural, N. and Iscan, M. (1996) Combined effects of ethanol and cigarette smoke on hepatic and pulmonary xenobiotic metabolizing enzymes in rats. Chem. Biol. Interact., 102, 155-167.

29. Vargas Nunes, S.O., Pizzo de Castro, M.R., Moreira, E.G., Guembarovski, R.L., Barbosa, D.S., Vargas, H.O., Piccoli de Melo, L.G., Bortolasci, C.C., Watanabe, M.A., Dodd, S., Berk, M. and Maes, M. (2015) Association of paraoxonase (PON)1 activity, glutathione S-transferase GST T1/M 1 and STin.2 polymorphisms with comorbidity of tobacco use disorder and mood disorders. Neurosci. Lett., 585, 132-137. 